\title{
Polymers for additive manufacturing: Collected contributions for MRS Communications
}

Jamie M. Messman and Daniel E. Bowen, Kansas City National Security Campus, DOE, Kansas City, MO, USA

Address all correspondence to Jamie M. Messman at jmessman@kcnsc.doe.gov

Published online 22 June 2021

Polymer Additive Manufacturing (PAM) is a remarkable custom manufacturing tool, which is in a broad transition from academia and hobbyist to industry. This transition is enabled by real possibilities, end use performance advantages, and favorable business cases in increasing numbers, especially where agility, customization, and complexity are required. While advances and improvements in established PAM processes and equipment, including fused filament fabrication, powder bed fusion, and digital light processing, are accelerating this transition, PAM equipment manufacturers are focused on the relatively narrow suite of proven materials. This gap, as well as that created by new PAM processes and equipment, create incredible opportunities for basic, fundamental, and applied research in pursuit of value added performance-structure-property-process (PSPP) relationships. As a result, this area of material science has witnessed growth in cutting-edge research and development, as well as the deployment of advanced manufacturing methods enabling rapid development and production.

This combination of advances in processes and materials is what brought PAM from rapid prototyping in the 1980s to what it is today. The combination of new additive processes and materials has spurred the increased use of additively manufactured parts in a growing number of applications, especially in aerospace, automotive, consumer products, energy, and medical/dental. PAM continues to flourish in a wide variety of low-volume, highly customizable product sectors, and the significant increase in academic involvement is helping to drive improved understanding of PSPP, which is further establishing broad usage and continued innovation. In this special issue of MRS Communications, contributions highlight novel materials, improved understanding of PAM processes, enhanced properties, and the scalability of PAM. 